\title{
A Rare Case of a Patient with Hypoplastic Left Heart Syndrome and Subsequent Congenital Long Qtc Syndrome \\ Putschoegl $\mathrm{A}^{1 *}$, Moran $\mathrm{AM}^{2}$ and Epstein $\mathrm{M}^{2}$
}

${ }^{1}$ Department of Internal Medicine and Pediatrics, Resident, Maine Medical Center, Portland, USA

${ }^{2}$ Department of Cardiology, Maine Medical Center, Portland, USA

\begin{abstract}
Hypoplastic left heart syndrome continues to be one of the most complicated and devastating of all the congenital heart defects. Despite advances in surgical palliation, patients continue to face a high percentage of morbidity and mortality. This syndrome can be associated with multiple other genetic conditions although we believe we have found the first case that is associated with a congenitally prolonged QTc. We will discuss this presentation and the importance of considering multiple syndromes in those with congenital heart disease.
\end{abstract}

Keywords: Hypoplastic left heart syndrome; Long QT syndrome; Chromosome 11

\section{Introduction}

Hypoplastic Left Heart Syndrome (HLHS) is a spectrum of leftheart obstructive lesions resulting in the underdevelopment of the mitral valve, left ventricle, left ventricular outflow tract, aortic valve and the aorta [1]. Therefore, the right ventricle is responsible for maintaining both the systemic and pulmonary circulation. Children typically undergo a three stage surgical palliation that successfully allows the child to live with a single, functional ventricle.

Long QT syndrome (LQTS) is characterized by abnormal QT interval prolongation and susceptibility for cardiac arrest and sudden death owing to a specific type of polymorphic ventricular tachycardia, torsades de pointes [2]. In the late 1990's, the first five LQTS genes were identified, all of which encoded proteins that form ion channels that underlie the cardiac action potential [2]. The patient in our case was found to have LQT-1, which is located on chromosome 11. General management of LQTS includes the prevention of torsades de pointes with the use of beta-blockers, implantable cardiac defibrillators and avoidance of any medication with the potential to prolong the QT.

\section{Case Report}

We report an 11-year-old female that was born with multiple cardiac defects including subaortic obstruction, mitral stenosis, hypoplastic aortic arch, hypoplastic left ventricle and coarctation of the aorta. Due to these multiple left sided defects, the patient was diagnosed with Shone's syndrome; however, the most significant was her hypoplastic left ventricle and aortic arch, which subsequently gave her the diagnosis of hypoplastic left heart syndrome. Therefore, here treatment was directed at the latter diagnosis and this is how her cardiac condition will be classified throughout this case report. She underwent the Norwood Stage I procedure as a first step towards a univentricular Fontan operation. One month later she required balloon dilation of a recurrent coarctation. Her recovery was complicated by a postoperative seizure that was treated with phenobarbital. Her EKG at that time showed right axis deviation with right atrial and ventricular hypertrophy. She was treated with digoxin, Lasix and baby aspirin.

Six months later the patient underwent a bi-directional Glenn. This was complicated by a paralyzed right hemi-diaphragm, which was complicated. She also had large venovenous collateral coiled because it resulted in significant cyanosis. On follow up, she had oxygen saturation at $72-76 \%$ and an EKG that revealed normal sinus rhythm with right ventricular hypertrophy. At three years of age the patient underwent a fenestrated Fontan repair. At follow up her oxygen saturation was $86 \%$ with no other concerning physical exam findings. She was treated with enalapril and aspirin. Two years later she had fenestration closure with a $4 \mathrm{~mm}$ Amplatz device without complication. Subsequently her aspirin was discontinued secondary to a familial platelet disorder (specifics unknown).

At six years of age the patient's EKG showed sinus bradycardia with junctional escape and some premature atrial beats and perhaps an atrial couplet. This was a new finding at this age. A 24-hour Holter monitor was placed that showed sinus rhythm with sinus bradycardia throughout. Over the following six months the patient experienced some chest pain and intermittent cyanosis. She had another 24 hour Holter monitor placed with no concerning findings. Her EKG's and 24 hour Holter monitoring over the next four years were only consistent for bradycardia with no associated symptoms.

At ten years of age the patient had an EKG at follow up that showed a prolonged QT interval at 460 milliseconds, which was abnormally long. In review of her old records, her QTc ranged anywhere from 410 - 500 upon manual measurement. Most of these measurements were around 440 milliseconds, which are considered normal (Table 1). The patient was referred to an electrophysiologist, who confirmed that she did indeed have a prolonged corrected QT interval. Testing was done on the patient's parents and brother, none of which had corrected QT prolongation. Despite no family history of long QT syndrome, the patient was treated as having congenital long QT and started on nadolol. Genetic testing was then performed showing that the patient was heterozygous for a published missense mutation in the KCNQ1 gene consistent with the autosomal dominant form of LQT-1 found on chromosome 11.

*Corresponding author: Adam Putschoegl, Department of Internal Medicine and Pediatrics, Resident, Maine Medical Center, 22 Bramhall St., Portland, ME 04102, 207-662-0111, USA, E-mail: putsca@mmc.org

Received October 17, 2013; Accepted November 06, 2013; Published November 13,2013

Citation: Putschoegl A, Moran AM, Epstein M (2013) A Rare Case of a Patient with Hypoplastic Left Heart Syndrome and Subsequent Congenital Long Qtc Syndrome. J Cardiovasc Dis Diagn 1: 132. doi:10.4172/2329-9517.1000132

Copyright: (c) 2013 Putschoegl A, et al. This is an open-access article distributed under the terms of the Creative Commons Attribution License, which permits unrestricted use, distribution, and reproduction in any medium, provided the original author and source are credited. 


\begin{tabular}{|c|c|c|}
\hline Age (years) & cQTc (milliseconds) & Normal vs. Abnormal \\
\hline $0-1$ & $400-500$ & Both \\
\hline 2 & 440 & Normal \\
\hline 3 & $410-500$ & Both \\
\hline 4 & 470 & Abnormal \\
\hline 5 & 440 & Normal \\
\hline 6 & 490 & Abnormal \\
\hline 7 & 470 & Abnormal \\
\hline 8 & 480 & Abnormal \\
\hline 9 & 440 & Normal \\
\hline 10 & 455 & Abnormal \\
\hline 11 & 460 & Abnormal \\
\hline
\end{tabular}

Table 1: Age vs. corrected QTc (cQTc).

\section{Discussion}

Hypoplastic left heart syndrome was first described in 1952 by Lev and was ultimately given this term by Noonan and Nadas. It accounts for $7.5 \%$ of the newborns with congenital heart disease [3]. HLHS accounts for $25 \%$ of cardiac deaths within the first week of life and $15 \%$ of cardiac deaths within the first month on life [3]. Longitudinal data from the Atlanta metropolitan region suggest that the incidence of HLHS, at 2.1/1000 live births, has changed little over the past 30 years [3].

The embryologic cause of HLHS remains to be fully understood. There is also little evidence to show that HLHS is genetically linked to one chromosome. This is not surprising given the fact that multiple anomalies are present in this disorder. In one study, however, there was found to be a link in chromosomes $10 \mathrm{q}$ and $6 \mathrm{q}$ [4]. Despite this finding, most neonates are born at term with no other non-cardiac anomalies in comparison to other infants with structural heart disease [3].

Norwood and others reported the first successful palliation of HLHS in a series of infants operated on between 1979 and 1981 [5]. Although these procedures have been refined, the essential elements include an atrial septectomy, anastomosis of the proximal pulmonary artery to the aorta with augmentation of the aortic arch and an aortopulmonary shunt [5]. Recent studies looking at survival for these patients has shown a decrease in mortality. A retrospective study from 1984- 1999 showed a one-year survival rate of $51 \%$ although over the last four years of that study, survival was increased to $71 \%$ [3]. More recently, a study by Photiadis et al. showed midterm survival ( 8 months) was excellent for patients with a stable preoperative condition and low Aristotle comprehensive score, reaching almost 90\% [6]. Otherwise, mid-term survival drops to $\sim 60 \%$. As a result, prenatal diagnosis and early referral (at birth) could be of the utmost importance.

To our knowledge this is the first case of combined HLHS and long QT syndrome. An extensive literature review was done, which showed no published cases of a combination of these two disorders. We were also curious if there were any published cases showing a correlation between any of the genes involved in LQTS and HLHS. We specifically looked at chromosome 11, since this was the location of the gene for LQTS involved in our case. This, again, resulted in no reported cases. We also looked into the relationship between chromosome 11 and other cardiac defects. This resulted in multiple anomalies including ventricular septal defects, coarctation of the aorta with aortic hypoplasia and faulty proteoglycan synthesis causing cardiac defects [7-9]. Through these finding, it does suggest that chromosome 11 has some genetic role in left sided anomalies although none specific to HLHS.

This case also proves important because of its possible implication for sudden, interstage death. Some factors associated with mortality between staged palliations include a restrictive atrial septal defect, older at the time of surgery, respiratory complications, neurologic events and arrhythmias. In one study, arrhythmias were associated with as many as four percent of interstage deaths $[6,10]$. It was unclear whether these arrhythmias were known prior to surgery or secondary to cardiac manipulation/ischemia. The child in our case could be considered lucky because her risk for arrhythmia did not cause her any undue morbidity or mortality despite her long QTc going undetected between stages of palliation.

\section{Conclusion}

We ultimately came to the conclusion that this patient was unfortunate enough to have two distinct cardiac entities that were completely unrelated in terms of their causal relationship. It is therefore always important to keep an open mind and consider other, separate diagnoses in all children with congenital heart disease with a specific focus on arrhythmias due to their high risk of sudden, interstage death.

\section{References}

1. Victoria L. Vetter (2006) Pediatric Cardiology, The Requisites in Pediatrics Mosby, Inc, Philadelphia, USA.

2. Allen HD, Driscoll DJ, Feltes TF, Shaddy RE (2008) Heart disease in infants children, and adolescents including the fetus and young adult. Wolters Kluwer Philadelphia, USA

3. Nichols DG, Ungerleider RM, Spevak PJ, Geeley WJ, Cameron DE, et al. (2006) Critical heart disease in infants and children. (2ndedn), Mosby, Inc Philadelphia, USA.

4. Hinton RB, Martin LJ, Rame-Gowda S, Tabangin ME, Cripe LH, et al. (2009) Hypoplastic left heart syndrome links to chromosomes $10 q$ and $6 q$ and is genetically related to bicuspid aortic valve. J Am Coll Cardiol 53: 1065-1071.

5. Mavroudis C, Backer CL (2003) Pediatric Cardiac surgery. ( $\left.4^{\text {th }} \mathrm{Edn}\right)$, Mosby, Inc, Philadelphia, USA

6. Photiadis J, Sinzobahamvya N, Haun C, Schneider M, Zartner P, et al. (2012) Does the shunt type determine mid-term outcome after Norwood operation. Eur J Cardiothorac Surg 42: 209-215.

7. Baasanjav S, Al-Gazali L, Hashiguchi T, Mizumoto S, Fischer B, et al. (2011) Faulty initiation of proteoglycan synthesis causes cardiac and joint defects. Am J Hum Genet 89: 15-27.

8. Palano GM, Licata F, Carpinato C, Sottile F, Sciuto R, et al. (2010) Coarctation of the aorta with aortic arch hypoplasia in newborn with partial trisomy $11 \mathrm{q}$ associated to $4 q$ interstitial deletion. Minerva Pediatr 62: 591-598.

9. Ye M, Coldren C, Liang X, Mattina T, Goldmuntz E, et al. (2010) Deletion of ETS-1, a gene in the Jacobsen syndrome critical region, causes ventricular septal defects and abnormal ventricular morphology in mice. Hum Mol Genet 19: 648-656.

10. Hehir DA, Dominguez TE, Ballweg JA, Ravishankar C, Marino BS, et al. (2008) Risk factors for interstage death after stage 1 reconstruction of hypoplastic left heart syndrome and variants. J Thorac Cardiovasc Surg 136: 94-99. 